\title{
OPSOCLONUS MYOCLONUS PRESENTING AS STATUS EPILEPTICUS
}

Two cases of non-epileptic opsoclonus presenting as status epilepticus are reported fom the John Radcliffe Hospital, Oxford, UK. Jerking of eyes and limbs were initially explained as suspected encephalitis, and the patients were treated for seizures with anticonvulsants and anesthetic intubation, but without benefit. EEGs showed no epileptic discharges. A diagnosis of opsoclonus myoclonus was made in both cases, and treatment with adrenocorticotropic hormone (40 IU/day) in one and prednisolone (4 mg/ $\mathrm{kg} /$ day) in the other resulted in rapid resolution of symptoms. No neoplasm or infectious agent was identified, and neither patient has relapsed or shown developmental delay. Video footage of both patients showing florid jerking suggestive of status epilepticus is presented on line. (Haden SV, McShane MA, Holt CM. Opsoclonus myoclonus: a non-epileptic movement disorder that may present as status epilepticus. Arch Dis Child 2009;94:897-899). (Respond: Dr Sarah V Haden, Community Paediatrics Department, Level LG 1, Children's Hospital, John Radcliffe Hospital, Headley Way, Oxford OX3 9DG, UK. E-mail: sarah.haden@orh.nhs.uk).

COMMENT. The electroencephalogram is indispensable in the distinction of nonepileptic paroxysmal disorders from epileptic seizures and in the diagnosis of nonconvulsive status epilepticus and an encephalopathic process. (Markand ON. Pearls, perils, and pitfalls in the use of the electroencephalogram. Semin Neurol 2003;23(1):7-46).

Outcome of opsoclonus-myoclonus studied in 11 patients at Children's Memorial Hospital, Chicago found that 9 of 10 treated with ACTH had recurrence of symptoms during a gradual withdrawal of $\mathrm{ACTH}$; prednisone in one patient was ineffective in controlling opsoclonus-myoclonus. Eight had developmental delay with motor incoordination and speech delay ( 7 with neuroblastoma and 1 without). Tumor removal did not improve symptoms. One of 8 with tumor and 2 of 3 with no tumor had normal neurologic development. (Hammer MS, Larsen MB, Stack CV. Pediatr Neurol 1995;13(1):21-24).

\section{INFANTILE CONVULSIONS AND RETINAL HEMORRHAGES}

The prevalence of retinal hemorrhages in infants presenting with convulsions was studied at Hospital Universitari Sant Joan de Deu, Barcelona, Spain. Of 389 children seen in the accident and emergency epartment with convulsions, 182 aged 15 days to 2 years were admitted with a first convulsion over a 2-year period (May 2004-May 2006), and 2 had retinal hemorrhages. All infants were examined within 72 hours of admission by an ophthalmologist using indirect ophthalmoscopy. Both infants with retinal hemorrhages were diagnosed with shaken baby syndrome. Convulsions alone are unlikely to cause retinal hemorrhages in children $<2$ years of age. (Curcoy AI, Trenchs V, Morales M, Serra A, Pineda M, Pou J. Do retinal hemorrhages occur in infants with convulsions? Arch Dis Child 2009;94:873-875). (Respond: Dr Ana Isabel Curcoy, Paseig Sant Joan de Deu, 2, 08950 Esplugues de Llobregat, Barcelona, Spain. E-mail: acurcoy@hsjdbcn.org).

COMMENT. A similar prospective study at Sackler School of Medicine, Tel Aviv University. Israel examined 153 children (aged 2 months to 2 years) in the ED after a 